\title{
Evolutionary life history theory as an organizing framework for cohort studies: Insights from the Cebu Longitudinal Health and Nutrition Survey
}

Christopher W Kuzawa ${ }^{1,2^{*}}$, Linda Adair ${ }^{3}$, Sonny A Bechayda ${ }^{4}$, Judith Rafaelita B Borja ${ }^{4}$, Delia B Carba $^{4}$, Paulita L Duazo ${ }^{4}$, Dan TA Eisenberg ${ }^{5}$, Alexander V. Georgiev ${ }^{6}$, Lee T Gettler ${ }^{7}$, Nanette R Lee $^{4}$, Elizabeth A Quinn ${ }^{8}$, Stacy Rosenbaum ${ }^{9}$, Julienne Rutherford ${ }^{10}$, Calen Ryan ${ }^{1}$, Thomas W McDade $^{1,2}$

${ }^{1}$ Department of Anthropology, Northwestern University

${ }^{2}$ Institute for Policy Research, Northwestern University

${ }^{3}$ Department of Nutrition and Carolina Population Center, University of North Carolina at Chapel Hill

${ }^{4}$ Office of Population Studies Foundation, University of San Carlos, Cebu Philippines

${ }^{5}$ Department of Anthropology and Center for Studies in Demography and Ecology, University of Washington

${ }^{6}$ School of Natural Sciences, Bangor University, Bangor, UK

${ }^{7}$ Department of Anthropology \& Eck Institute for Global Health, University of Notre Dame

${ }^{8}$ Department of Anthropology, Washington University in St Louis

${ }^{9}$ Department of Anthropology, University of Michigan

${ }^{10}$ Department of Women, Children, and Family Health Science, College of Nursing, University of Illinois at Chicago

For special issue of Annals of Human Biology focused on Cohort Studies

Running head: Testing life history theory in the Cebu cohort

*Correspondence: kuzawa@ northwestern.edu 


\begin{abstract}
By tracking a group of individuals through time, cohort studies provide fundamental insights into the developmental time course and causes of health and disease. Evolutionary life history theory seeks to explain patterns of growth, development, reproduction and senescence, and inspires a range of hypotheses that are testable using the longitudinal data from cohort studies. Here we review two decades of life history theory-motivated work conducted in collaboration with the Cebu Longitudinal Health and Nutrition Survey (CLHNS), a birth cohort study that enrolled more than 3000 pregnant women in the Philippines in 1983 and has since followed these women, their offspring and grandoffspring. This work has provided evidence that reproduction carries "costs" to cellular maintenance functions, potentially speeding senescence, and revealed an unusual form of genetic plasticity in which the length of telomeres inherited across generations is influenced by reproductive timing in paternal ancestors. Men in Cebu experience hormonal and behavioral changes in conjunction with changes in relationship and fatherhood status that are consistent with predictions based upon other species that practice biparental care. The theoretical expectation that early life cues of mortality or environmental unpredictability will motivate a "fast" life history strategy are confirmed for behavioral components of reproductive decision making, but not for maturational tempo, while our work points to a broader capacity for early life developmental calibration of systems like immunity, reproductive biology and metabolism. Our CLHNS findings illustrate the power of life history theory as an integrative, lifecourse framework to guide longitudinal studies of human populations.
\end{abstract}


Most adult humans alive today will, in time, die from a chronic disease that develops in response to a lifetime of exposure to a diverse set of environments and experiences. Many conditions are influenced by the chronicity of specific exposures across the lifecycle, whether beneficial or harmful. Critical periods are increasingly understood as heightening sensitivity to specific classes of exposures and experiences in defined developmental windows, thus linking early nutrition, stress and other factors with later biology and health (Barker, 1990, Hanson and Gluckman, 2011). By following a group of individuals longitudinally, cohort studies are ideally suited to addressing these temporal and developmental dimensions of phenotypes and disease emergence, and such studies have provided foundational knowledge about the causes of some of the globally most pressing public health issues. The Framingham heart study that began in 1948 has provided fundamental insights into the role of biological processes like blood pressure and lipid metabolism in the development of atherosclerosis and coronary heart disease (Ho et al., 1993). The Whitehall study similarly helped lay the foundations for contemporary understandings of the role of social class as a driver of health inequality within society (Marmot et al., 1991). The Dunedin Longitudinal Study has provided key insights into the long term psychological and health effects of early psychosocial adversity (Poulton et al., 2015). Cohort studies in countries like the Gambia (Hennig et al., 2017), Guatemala (Stein et al., 2008), South Africa (Cameron et al., 1998, Richter et al., 2007), the Philippines (Adair et al., 2011), Bangladesh (Arifeen et al., 2018) and Jamaica (Walker et al., 2005) have clarified the influence of factors like nutrition, infectious disease, and infant feeding practices on infancy and childhood growth and development, and traced the long-term impacts of these early experiences on human economic and health outcomes measured in adulthood. 
The longitudinal dimension of cohort studies provides powerful insights into the time course of disease development, and the types and timings of exposures that increase or decrease this risk. What is often less clear, and traditionally has rarely been addressed by such studies, is why our bodies and behavior tend to respond predictably to our experiences. Longitudinal studies not only reveal the power of chronic experience and of sensitive or critical periods, but demonstrate that our biology and behaviors tend to co-vary and flex in predictable ways as environments change. As one example, it is intuitive that early severe stressors might lead to cognitive impairment, but it is less clear why individuals who experience such stress also tend to begin their reproductive careers earlier or have larger completed family sizes (Nettle et al., 2011, Wilson and Daly, 1997). In turn, parity has been shown to predict an accelerated pace of late life functional decline, aging and mortality (Jasienska, 2009, Doblhammer and Oeppen, 2003). Findings such as these illustrate how the full impact of our experience on health and wellbeing must be understood as a series of changes that cascade through the lifecycle. The coordinated nature of these changes also hints at an underlying strategy that has a capacity to adjust in response to the physical and social environments that we experience.

Life history theory (LHT) is the branch of evolutionary theory that seeks to understand the evolution of species variation in life cycles, and also how and why they vary across individuals of the same species (Charnov, 1991, Kuzawa and Bragg, 2012, Hill and Kaplan, 1999). For the purposes of human biological investigations using cohorts, life history theory provides a framework for formulating a priori, testable hypotheses regarding an organism's metabolism and pattern of energy allocation to the body's various functions, and focuses attention on the finite nature of resources and the resultant trade-offs that bind traits into patterns of co-variation. It also inspires hypotheses for how behaviors or experiences early in life might 
impact various outcomes measured in adulthood, including such traits as reproductive biology, behavior, immunity and the pace of senescence and functional decline. Life history theory thus provides a useful scaffold for our investigations of human biology and its unfolding across the lifecourse and even across generations.

In this paper, we first briefly review some of the core organizing principles of LHT as they have been used to illuminate the evolution of interspecific variation in life cycle characteristics like growth rate, body size and lifespan. The scaling of metabolic expenditure on an individual's body mass places fundamental constraints on the energy and other metabolic resources available to meet the body's many functions, leading unavoidably to trade-offs (Urlacher et al., 2019). With the body's energy budget set by body size, the optimal pattern of allocation across functions is driven in part by ambient levels of unavoidable mortality, which determine the likely future returns on investing in somatic durability and lifespan extension. After this review, we will discuss our use of life history principles as an organizing framework for a subset of our investigations of the Cebu Longitudinal Health and Nutrition Survey, a birth cohort that began in 1983 and has since followed a large sample of women, their offspring and grandoffspring living in metropolitan Cebu, Philippines (Adair et al., 2011). We will discuss some of our tests of life history principles, focusing on questions like the links between reproduction and markers of aging, the role of telomeres as an intergenerational life history cue, hormones in the coordination of male life history and reproductive strategy, and developmental calibration of immune development and life history trade-offs. We will conclude by considering the limitations of LHT for use in cohort studies, and argue that they are primarily a result of our limited understanding of the functional significance of many the biomarkers that we routinely collect, which are targeted for predictive relationships with biological dysfunction and disease. 
Life history primer: metabolic scaling, trade-offs and unavoidable mortality

Organisms vary widely in characteristics like body size, growth rate, age at first reproduction, reproductive rate and lifespan. These traits tend to co-vary such that species that are larger as adults also tend to have an extended period of relatively slower growth, invest a smaller fraction of their metabolic resources in offspring (reproduction), and have longer lifespans. Life history theory (LHT) is the branch of evolutionary theory that seeks to explain the evolution of life cycles, and has helped shed light on these predictable patterns of trait covariation (Stearns, 1992).

The scaling of an organism's energy expenditure on body size, as reflected in the classic “three-quarter" scaling of a species' energy expenditure on body mass (Kleiber, 1932), provides a useful starting point for considering the factors that constrain the evolution of life histories. One model to explain this regularity, proposed by West and colleagues (1999), posits that an organism's metabolic expenditure is limited by its ability to distribute resources. Animal circulatory systems are organized to deliver blood, enriched with oxygen, energy and other nutrients, from a single, large vessel (the aorta) to capillaries that service individual cells throughout the body. West and colleagues note that the strategy for distributing resources within a given mass that minimizes hydrodynamic resistance within the system is a fractal network in which large vessels branch into smaller vessels, with nested vascular branching continuing until individual cells are reached. The authors show that such a fractal distribution network leads to 0.75 scaling of energy expenditure on mass, irrespective of the size of the organism or system (West et al., 2002), thus potentially helping explain metabolic scaling from first principles of physics. 
Metabolic scaling shows that the energy available to an organism of a particular size is finite, which implies that any expenditure of a metabolic resource (e.g. a gram of glucose, an amino acid or molecule of ATP) on one function leaves fewer resources available to be allocated to the body's other functions and needs. As demonstrated empirically in humans, constraints on energy budgets lead unavoidably to functional trade-offs (Kuzawa et al., 2014, Urlacher et al., 2019), which the metabolic and physiologic architecture of the human body has been designed to balance. This concept of trade-offs is central to LHT-focused analyses, which emphasize both immediate or concurrent resource trade-offs, as illustrated for instance by the fact that testosterone boosts muscle anabolism (in males a form of mating effort) but at a cost to immunity (a maintenance function)(Muehlenbein and Bribiescas, 2005), or lagged trade-offs in which expenditures at one point in the lifecycle influence future phenotypes.

As a result of finite energy, complex multi-cellular organisms face a fundamental decision: what fraction of their metabolic resources to invest in growing new tissue (becoming bigger during growth, or growing the tissue of the next generation during reproduction) - versus repairing and maintaining the tissues and organs that are already present (extending lifespan). The idea that reproduction carries costs to survival, thus reducing future reproduction, is a longstanding assumption in evolutionary biology (Williams, 1966), and is a key inspiration for the "disposable soma" model for the evolution of senescence (Kirkwood and Rose, 1991). The disposable soma posits a trade-off between reproduction and maintenance expenditures, such that species that invest a greater fraction of their metabolic budget in reproduction have fewer resources to invest in functions that help maintain durability and function of the body and brain into later life. Although the disposable soma has gained support from some animal models (Bouwhuis et al., 2010), applicability to human senescence has been mixed; for instance, in some 
historical data, lifespan is inversely related to fertility (Westendorp and Kirkwood, 1998), while in others the two measures are positively related (see Mitteldorf, 2010). Although the reason for these inconsistencies is uncertain, human studies have shown that trade-offs can be obscured when individuals vary widely in nutritional sufficiency or resource access (Hill and Hurtado, 1996). It has also been noted that the costs of reproduction may be minimized in some recent high socioeconomic cohorts owing to reduced reproductive costs associated with the use of infant formula (Jasienska, 2009).

A second assumption of many LHT frameworks is that unavoidable or "extrinsic" mortality, such as is approximated by predation or unpredictable features of the environment that impact survival, influences the optimal strategy of partitioning resources across these various functions, while also helping determine the optimal age at maturity. According to this principle, mice mature earlier than elephants, and attain a much smaller final size, in large part because they are subjected to high rates of predation, making it risky to delay maturity further. Once mature, they also invest a greater fraction of the body's metabolism to reproducing now (and thus, less to maintenance effort), given that chances of surviving to reproduce in the future are reduced by high unavoidable mortality. Another way of stating this is that the high likelihood of predation among mice makes them pessimistic about their futures, forcing them to adopt a "live fast, die young" strategy.

Because organisms often face substantial variability in their environments and experiences, natural selection generally does not set an individual's LH trajectory genetically, but instead allows some degree of flexibility in the form of developmental and phenotypic plasticity (Stearns and Koella, 1986, Kuzawa and Bragg, 2012). Most notably, experience of local cues reflecting mortality or environmental unpredictability have been predicted to motivate 
a "fast" life history strategy in which reproduction is initiated earlier and the rate of investment in each offspring (as reflected for instance in birth size, duration of breastfeeding or interbirth interval) is reduced. This increase in reproductive expenditure in turn is predicted to increase the costs of reproduction and accelerate functional decline and senescence. Other capacities to adjust life history priorities play out in an ongoing fashion across the lifecycle, such as through the dynamic effects of hormones that mediate trade-offs between functions like reproduction and immunity.

\section{Testing LHT with the CLHNS}

During the past several decades we and our collaborators and students have used principles from LHT to help organize our work on the Cebu Longitudinal Health and Nutrition Survey (CLHNS). The CLHNS was initiated in 1983 by a team that included Barry Popkin and Wilhelm Flieger, with the recruitment of 3327 women who were pregnant (for overview of study design and timeline please see Adair et al., 2011). Their offspring were followed after birth, and surveys during the first two years recorded information on infant feeding, including breastfeeding and supplemental foods, maternal reports of infectious disease symptoms (e.g. diarrhea or respiratory infections), and length and weight growth. Linda Adair later spearheaded an effort to relocate the original birth cohort in 1991, when they were an average of 8.5 years old. The original mothers, their offspring, and now the grandoffspring, have been followed periodically to the present, representing more than 35 years of data collection across three generations (Adair et al., 2011, Eisenberg et al., 2019). Standardized modules on diet, income, health, sexual activity and a wide range of other individual, household and neighborhood characteristics were recorded regularly until the cohort was in young adulthood. Surveys since 2005 have focused on longitudinal follow-up of males to measure salivary hormones and 
reproductive ecology (Rosenbaum et al., 2018), while adult females were tracked to locate new pregnancies and to obtain information during the pregnancy and on birth outcomes of offspring (Kuzawa et al., 2017). Other recent surveys have focused on aging in the mothers of the CLHNS birth cohort, and on adult cognition and wellbeing in the offspring. These data have provided a rich resource to address a wide array of questions of interest to fields like economics, sociology and demography. In addition, our own anthropologically-oriented work has harnessed these data to address many questions in population biology, including a subset inspired by evolutionary LHT. Here we will review some of this work as it applies to questions around the biology of aging, male reproductive ecology and life history, the role of early experiences as cues that set life history strategy, and the developmental ecology of human immunity.

Insights into the biology of aging: Testing the trade-off between reproduction and somatic maintenance

The trade-off between reproduction and somatic maintenance that is central to LHT inspired models of aging can be understood as a decision between investing energy in current reproduction versus investing in functions that promote survival and thereby allow reproduction in the future. Costs of reproduction are central among trade-offs in LHT, and as noted, are also fundamental to the disposable soma model for the evolution of senescence. In Cebu we've tested for costs of reproduction using two complementary measures of biological aging: telomere length and epigenetic age acceleration (Ryan et al., 2018). Telomeres are repeating DNA sequences that cap the ends of chromosomes and are required for normal cell replication. With each replication telomere length is shortened and cells undergo programmed cell death (apoptosis) when a critically short telomere length is reached (Eisenberg, 2011). Epigenetic age 
is a measure of molecular aging based on predictable changes to DNA methylation in the genome. While epigenetic age is closely tied to chronological age, accelerated epigenetic age relative to chronologic age is associated with elevated mortality risk and a range of adverse health outcomes independent of other risk factors.

Using telomere length and epigenetic age as measures of cellular aging and predictors of mortality and health, we found evidence that women's cells 'age' with each additional pregnancy (Fig. 1). This effect was not attributable to genetic variation or to a range of social factors, such as urbanicity, parental education, household assets, or income, supporting the theorized tradeoff between reproduction and bodily maintenance at the cellular level (Ryan et al., 2018). Moreover, neither measure predicted the number of pregnancies over the subsequent four years, implying that reproductive effort is likely driving cellular aging in these women rather than increasing in response to an intrinsically accelerated pace of aging more generally. These findings support of the assumption that reproduction carries costs among women. In an unrelated earlier analysis, we also found evidence that anti-mullerian hormone, a marker that reflects follicular reserve, was reduced in relation to parity, also potentially pointing at a cost of reproduction (Bragg et al., 2012). What is less clear is whether these relationships will still be detectable at older ages when cellular senescence leads to functional decline (or in the case of anti-mullerian hormone, as declining follicular counts impairs fecundity).

The paternal age at conception effect on telomere length as a form of genetic plasticity in life history allocation

While increasing reproductive effort during early adulthood may accelerate blood telomere shortening in females, the dynamics of telomere length in the germ line allow for a unique form of intergenerational genetic plasticity that could link male reproduction to patterns 
of life history allocation in future generations. Telomere length shortens with age in most human tissues, but spermatocyte telomere length increases with age (Eisenberg and Kuzawa, 2018), which appears to be driven by the continuous production of sperm in the presence of high levels of testicular telomerase (an enzyme that lengthens telomeres). Correspondingly, offspring of older fathers have longer telomere length (Eisenberg and Kuzawa, 2018). Our work with the CLHNS demonstrated that this effect of paternal age at conception persists cumulatively across at least two generations - that is, the age at which paternal grandfathers sired fathers and maternal grandfathers sired mothers predicts the grandchild's telomere length additively and independently to the effect of their own father's age on their telomere length (Eisenberg et al., 2012, Eisenberg et al., 2019).

These findings are important because they suggest that telomere length in any given generation is partially a reflection of the average age of reproduction among recent generations of male ancestors. Because the age at reproduction is a key life history parameter, this integrative quality of the paternal age at conception effect hints at a capacity to calibrate a dimension of maintenance expenditure in response to a signal of the likely ages of reproduction in that population (for a similar example involving nutrition see Kuzawa, 2005). Because longer telomere length allows cells to replace themselves more readily, longer telomere length is implicated as a cause of improved immune function, better wound healing, and improved cardiovascular function (likely due to improved blood vessel repairs). All of these cell replication-dependent processes are likely to be energetically expensive and from a LHT perspective longer inherited TL may thus be viewed as a cause of increased maintenance effort (Eisenberg, 2011). Increasing allocation to somatic maintenance may thus be an adaptive shift in 
allocation priorities in demographic and cultural settings in which reproduction typically occurs later in life.

Because telomeres are DNA, the telomerase-driven changes in sperm telomere length that accrue with paternal age are highly heritable, which underlies the paternal age at conception effect. Emerging evidence suggests that sperm telomere length could be modified by other environmental or behavioral factors aside from male age, and these changes should similarly be highly heritable. In Cebu, we find that the offspring of men with shorter leg length tend to have longer telomeres. Although mechanistic explanations for this finding remain tentative, it is notable that short leg length — a measure of early developmental adversity— has been associated elsewhere with earlier puberty (Schooling et al., 2008), perhaps reflecting the maturationaccelerating effect of early growth deficits followed by catch-up growth (Gluckman and Hanson, 2006). Because individuals who experience puberty earlier likely also begin increasing their sperm telomere length at a younger age, the physiology of earlier puberty could increase the rate of age-related increase in sperm telomere length (Eisenberg et al., in press). This is particularly intriguing as age at puberty is influenced by nutritional availability (Bribiescas, 2006; p.111), including among the younger (cohort) generation at Cebu (see below). The general take away is that the telomere lengths that men pass on to their offspring might be adjusted not only in response to the age at reproduction, but also to their childhood nutritional circumstances and growth dynamics - paralleling intergenerational effects on other systems observed along the matriline (Kuzawa and Quinn, 2009).

Relationships, fatherhood and male reproductive effort

Across cultures, men provide varying levels and types of parental care and investment (Hewlett and Macfarlan, 2010, Lamb, 2004, Marlowe, 2000, Gray and Anderson, 2010, Gettler, 
2016). While this most commonly takes the form of providing resources, depending on the cultural context and individual family setting, men may regularly be involved in direct childcare (Hewlett, 1991, Winking et al., 2009, Mattison et al., 2014, Boyette et al., 2018, Rosenbaum et al. in review, Alvergne et al., 2009, Anderson et al., 2007). This is a vast departure from the behavior of most of other mammal species, a taxonomic class in which males provide paternal care in roughly 5\% of species (Lukas and Clutton-Brock, 2013, Kleiman and Malcolm, 1981). The rarity of this behavior, along with the importance of cooperative care-including paternal care - to the suite of life history traits that make humans unique, has generated great interest in whether physiological and behavioral expressions of mating-parenting tradeoffs in humans are similar to those of other species.

Though it is unusual in mammals, bi-parental cooperation in caring for young is quite common in many seasonally breeding bird species. An extensive body of work has demonstrated that male birds' testosterone rises during the time of year when males are competing for mates and defending their territories, but falls dramatically once it is time to care for nestlings (Wingfield et al., 1990). Across vertebrate species, testosterone commonly enhances mating-related somatic investment (e.g. skeletal muscle mass; ornamentation), dampens the fear response, and increases competitive-aggressive tendencies, all of which are desirable traits when fending off competitors. These tendencies however are largely incompatible with the very different demands that direct care and male-female cooperation involve (Muller, 2017, Gray et al., 2017, Gettler et al., 2014b). The physiological switch male birds experience presumably helps ensure that they have the physical capacity to deal with intraspecific challengers when necessary, but also that they are behaviorally capable of taking care of young once they arrive. 
This 'challenge hypothesis' aligns with the basic tenets of LHT because of its emphasis on the biological mechanisms that underpin the allocation of limited resources to competing demands. It has become the dominant framework for understanding the physiological trade-offs males make between mating and parenting effort in a much wider assortment of organisms (Wingfield, 2017, Grebe et al., 2019). The CLHNS cohort has provided a unique opportunity to test the applicability of the challenge hypothesis in humans, and to explore the role of endocrine change as a coordinator of men's changing social roles across adulthood. Though prior studies had observed that fathers had lower testosterone than non-fathers, it was unclear whether fatherhood caused a reduction in testosterone, as seen in birds, or alternatively, whether men with lower testosterone might be more likely to end up in stable relationships and become fathers (Gray et al., 2002, Kuzawa et al., 2009). In Cebu, testosterone predicts muscle and strength in early adulthood, consistent with the hormone's expected role in promoting costly components of mating effort (Gettler et al., 2010), although there was no evidence for testosterone's proposed suppressive effects on immunity (Gettler et al., 2014a). We also found that single non-partnered men with high testosterone at baseline ( 21 years of age) were more likely to have entered a stable relationship and become a father during a 4.5-year follow up period. The newlypartnered, new fathers then experienced large decreases in testosterone concentrations to end up with lower testosterone than men who remained single (Gettler et al., 2011a). Furthermore, the fathers who engaged in more direct care had lower testosterone than less involved fathers (Gettler et al., 2011a). These critical findings demonstrated the applicability of the general concepts of the challenge hypothesis framework to humans.

Other CLHNS work has further illuminated the physiological underpinnings of humans' remarkably flexible mating and parenting strategies. For example, parenting status is associated 
with lower testosterone not only in men, but also in women (Kuzawa et al., 2010a), and in fathers specific partnering and parenting behaviors can mediate the relationship between paternal status and a man's testosterone (Gettler et al., 2012b, Gettler et al., 2013). Moreover, we also found that other hormones (e.g. cortisol, prolactin) differ between CLHNS fathers and nonfathers, likely reflecting shifting priorities across life history transitions (Gettler et al., 2012a, Gettler et al., 2011b). Although there is little evidence that a common polymorphism in the androgen receptor alters regulation of testosterone production in the sample (Ryan et al., 2017), we did find that this polymorphism interacted with testosterone to influence traits like pairbond stability (Gettler et al., 2017). Additionally, in contrast to the predictions of the challenge hypothesis, men's testosterone does not appear to be closely tied to at least some measures of mating effort (Rosenbaum et al., 2018, Gettler et al., 2019). These results highlight the many nuances of human mating-parenting tradeoffs. Our current work is focused on understanding how developmental experience influences later-life psychobiological profiles (e.g. Sarma et al., 2018, Gettler et al. in prep), and the broader caretaking context in which male parenting effort occurs in Cebu (Rosenbaum et al. in review).

The role of early environmental cues as influences on life history strategy

Beyond the dynamic role of hormones in mediating trade-offs across the lifecycle, LHT also leads to an expectation that experiences early in development serve as informative cues that allow a calibration of the trajectory of development and life history strategy. Consistent with this concept, an extensive literature, much of it focused in psychology, provides evidence that individuals exposed to early life cues of mortality or environmental unpredictability tend to adopt features of a "fast" life history strategy, including traits like an earlier age at maturity, earlier onset of first reproduction, greater likelihood of risk taking and a shortened interbirth 
interval (Wilson and Daly, 1997, Belsky et al., 1991, Ellis et al., 2009). Our CLHNS findings provide partial support for these expectations. Although we have yet to explore these specific relationships in females, we find that males who experienced early life cues of mortality or environmental harshness have sex for the first time and become fathers at an earlier age (Fig. 2; Gettler et al., 2015). However, counter to the expectations of past evolutionary psychology oriented studies in other populations, in both males and females early cues of harshness predict delayed sexual maturity, while - consistent with most past work in other lower resources settings (e.g. Eveleth and Tanner, 1990)—multiple measures of favorable nutrition were strong predictors of maturational acceleration (Gettler et al., 2015, Kyweluk et al., 2018).

This set of findings confirms the long-appreciated role of nutrition (and more proximately, growth rate) as a fundamental constraint on timing of maturity (Eveleth and Tanner, 1990), while sexual behaviors may be largely uncoupled from physical maturity and thus influenced independently by cues of mortality or environmental harshness (Kuzawa and Bragg, 2012). It is notable that most past evidence for maturational acceleration in high stress environments has come from populations in which inequality, and the maturity-accelerating effects of overweight and obesity, are common, raising doubt about the evolutionary relevance of those findings (Kyweluk et al., 2018).

We have additional evidence that favorable — not stressful— nutritional environments are key to early physical maturity among study participants. Following earlier work in the cohort linking birth proportions to menarcheal timing among cohort females (Adair, 2001), we reported that rapid late-infancy weight gain, which in this population is an indicator of nutritional adequacy and a reduced burden of infancy infections, disproportionately impacts male adult phenotypes, as reflected in larger adult size, lean mass, muscle, grip strength and heightened 
testicular sensitivity to luteinizing hormone, leading to higher adult testosterone (Kuzawa et al., 2010b). The age when rapid weight gain has these long-term predictive relationships is specific to the first half year of postnatal life, which corresponds with the "mini puberty" of temporarily high $\mathrm{T}$ production that newborn boys experience. The finding that adult males who grew rapidly during this period also reported having more recent sex, had more lifetime sex partners, and an earlier age at first sex, suggesting potentially broad impacts of nutrition and endocrine regulation during this narrow early developmental window. These findings thus support the hypothesis that the mini puberty could serve as a critical or sensitive period for males, when nutrition and growth are predictive of subsequent growth trajectories and life history, including effects on regulation of testicular hormone production (Kuzawa, 2007). In Cebu, we find modest evidence that rapid growth during this window is associated with a more androgenic $2 \mathrm{D}: 4 \mathrm{D}$ ratio (the so called "digit ratio"), hinting that this ratio reflects not only exposure to testosterone during the prenatal period, as typically assumed, but possibly also to levels of the hormone during the early postnatal months (Georgiev et al., 2017). Growth during this window was found not to be a strong predictor of female outcomes, consistent with this being a critical period specific to male development (Kuzawa et al., 2010b). What is less clear at present is whether there are critical periods that are specific to females, or during which nutrition or growth processes have particularly strong effects on adult reproductive effort, as would be predicted to impact outcomes like offspring birth size.

Gestation and lactation are increasingly understood as an additional source of cues that convey information about local ecologies, and thus might allow calibration of life history strategy in offspring (Kuzawa and Quinn, 2009), and our work with the CLHNS has also explored these links. During pregnancy, maternal metabolism undergoes major shifts to help 
facilitate delivery of nutritional resources to the fetus, including a late gestation rise in glucose and triglycerides (Fried et al., 2017). In a sample of CLHNS participants, among whom birth weights are relatively light by comparative standards, we find that placentas $(n=22)$ are disproportionately small and efficient, producing more fetal mass per unit placental mass than is typically the case in more affluent populations; this increased efficiency of placental nutrient transport may be an important mechanism to buffer the fetus from maternal chronic undernutrition (Rutherford et al., 2019). In pregnant women in the sample, fasting glucose is a stronger predictor of birth weight in males compared to females (Fried et al., 2017), which parallels evidence for a similar male-biased effect of maternal cortisol during pregnancy on offspring birth weight (Thayer et al., 2012). These findings suggest that male offspring may place higher demands for resources on maternal metabolism and have heightened sensitivity as a result. We have speculated that these findings could help explain why measures of the gestational environment, such as birth weight or prematurity, have stronger relationships with cardiovascular risk factors, including adolescent lipid profiles (Kuzawa and Adair, 2003), blood pressure (Adair et al., 2001) and adult diurnal cortisol levels in this cohort (Lee et al., 2014). In contrast, there is no evidence that the composition of breast milk, or frequency of suckling, varies in relation to offspring sex among young mothers in the sample (Quinn, 2013). However, metabolic hormones in maternal milk vary in relation to the mother's body composition, and predict variation in offspring growth rate (Quinn et al., 2015, Anderson et al., 2016), possibly hinting at a capacity to set aspects of life history trajectory in response to measures of ecological quality such as the mother's chronic energy status.

\section{Life history tradeoffs and the developmental ecology of human immunity}


As noted above, life history theory underscores the importance of maintenance to survival, and in shaping life history tradeoffs and allocation strategies. However, with the exception of research on aging, most studies applying a LHT framework focus on issues related to reproduction and growth, with relatively little attention given to investments in the preservation and repair of the soma. Immunity plays central roles in host defense and repair, and therefore provides opportunities to operationalize a major component of maintenance effort (McDade, 2003). With the addition of several immune markers, beginning in 1999 and continuing today, Cebu data have made important contributions to our understanding of the developmental ecology of human immunity.

Investments in immune defenses are costly, and resources allocated to immunity are therefore not available for other developmental or physiological processes (McDade, 2003, McDade, 2005). A life history approach underscores that tradeoffs are therefore inevitable, and that investments in immunity are likely to be shaped by the availability of nutritional resources. Furthermore, one might hypothesize that nutrition early in development, during the establishment and rapid expansion of immune tissues and cells, will have disproportionate and lasting impact to the extent that current nutritional conditions forecast future environmental quality.

Using data from the 1998 survey, we showed that individuals born small-for-gestational age were less likely to respond to typhoid vaccination in adolescence (McDade et al., 2001). This study was important in that it was the first to demonstrate a long-term effect of prenatal undernutrition on immune function in humans, whereas prior work motivated by the "Barker hypothesis" was focusing primarily on cardiovascular and metabolic outcomes. Subsequently, we have shown that prenatal undernutrition is associated with reduced thymic hormone 
production in adolescence, as well as a lower proportion of B lymphocytes and CD4 helper T lymphocytes in young adulthood (McDade et al., 2018, McDade et al., 2001). Overall, this pattern of results suggests that prenatal undernutrition leads to reduced investment in key aspects of human immunity.

Life history theory provides a theoretical framework for understanding when, and why, low nutrition environments should reduce long term investments in aspects of immunity. This framework also encourages us to move beyond the prevailing emphasis on birth weight and consider other ecological inputs that might be important determinants of allocation decisions related to maintenance effort. The intensity of pathogen exposure is a likely candidate: the immune system is primarily responsible for protecting us against infectious disease, yet immune defenses are costly. Therefore, one might hypothesize that—all things being equal—investments in immunity will be higher in environments with higher levels of pathogen exposure. We tested this hypothesis in Cebu, taking advantage of one of the study's unique features: multiple measures of sanitation, microbial exposure, and infectious disease symptoms in infancy. We find that higher levels of infectious exposure in infancy are associated with a more robust antibody response to typhoid vaccination, and marginally higher proportions of CD4 and CD8 T lymphocytes in young adulthood (McDade et al., 2001, McDade et al., 2018).

Interestingly, we have also discovered that higher birth weight and higher levels of microbial exposure in infancy are associated with lower concentrations of C-reactive protein (CRP), an endpoint measure of systemic inflammation (McDade et al., 2010). A subsequent analysis identified DNA methylation as a potential mechanism linking early exposures and the regulation of inflammation in young adulthood (McDade et al., 2017). While these results may seem to run counter to the hypothesis that the body should prioritize investment in immunity 
when infectious disease risk is high (when payoffs are high) and/or when nutrition is abundant (when costs/trade-offs are minimized), they remind us that immunity is comprised of multiple subsystems of defense. No single measure can provide an indication of overall investment in immunity, and tradeoffs across subsystems of immune function are likely based on the relative costs and benefits of each.

To further elaborate this idea, we recently proposed, and tested, an "immunocalibration" hypothesis to explain variation in patterns of investment in innate (non-specific) and acquired (specific) immunity (McDade et al., 2016). While both subsystems play important, and complementary, roles in host defense, their functional profiles and associated costs are distinct. The development of acquired immunity is a time-dependent, antigen-driven process with relatively high upfront costs, but the costs of maintaining and activating specific defenses upon secondary antigen exposures are relatively low. Innate immunity provides rapid, generalized responses to novel (as well as secondary) pathogens that impose lower upfront developmental costs, but higher operating costs - including damage to host tissues. We therefore might expect that relative levels of investment in innate vs. acquired immune defenses will be optimized in response to local ecological cues that motivate "fast" vs. "slow" life history strategies, along the lines of our explorations of similar questions predicting timing and intensity of reproductive investments discussed above.

To test this idea, we hypothesized that abundant nutritional resources, higher levels of pathogen exposure, and low signals of extrinsic mortality risk would favor relatively higher levels of investment in acquired immunity. Using CRP as an indicator of innate immunity, and antibodies against the Epstein-Barr virus as an indirect measure of an aspect of cell-mediated, acquired immunity, we found general support for these predictions among the Cebu cohort in 
young adulthood (Georgiev et al., 2016). Additional tests with more in-depth measures of immunity are needed, but these findings—as well those described above — highlight the value of life history theory for providing an explanatory framework that organizes prior empirical findings and generates novel hypotheses regarding the impact of developmental environments on human immunity.

\section{Discussion}

Working with the CLHNS, we have used LHT to frame a wide array of hypotheses, which we have explored using the lifetime of longitudinal data available for study participants. Our efforts are by no means complete, and there are obvious gaps in our analyses to date. Perhaps most notably, we know more about male than female life history, in part because of the challenges of studying female reproductive endocrinology in a setting in which participants are distributed across urban and rural areas of a large metropolitan area of 3 million people, and in which the repeat sample collections typically needed to accurately characterize female reproductive function are not feasible. We find support for many key LHT assumptions, such as that reproduction carries "costs", and that cues of unstable or dangerous environments motivate an accelerated onset of first reproduction, along with a shift to an immune system oriented around defending against immediate challenges. Patterns of adult male reproductive behavior and biology unfold as predicted by observations in other species with similar mating systems. The timing and age of reproduction, in turn, appears to have intergenerational consequences for patterns of life history allocation in the next generation, manifesting as inheritance of longer telomeres (and we can surmise, enhanced allocation to lifespan-extending maintenance effort) when multiple recent generations of male ancestors have conceived at older average ages. 
Although a useful framework, there are notable challenges to testing LHT predictions in longitudinal human cohort studies. At a general level, much of the theoretical foundations of LHT were developed to explain between-species variation in life histories. Although these principles have been used fruitfully to gain insights into human developmental plasticity (Kuzawa and Bragg, 2012), they may not always translate to helping explain within-species variation (Stearns and Rodrigues, in press). As we discuss in detail elsewhere (Kyweluk et al., 2018), the important role of extrinsic mortality as a predictor of timing of maturity across species (Charnov, 1991) may not apply to within-species plasticity, in which factors like nutritional sufficiency may play a more central role.

The idea that finite energy leads to functional trade-offs is a core assumption of LHT, for these link functions to one another in predicted patterns of co-variation. Methodologically, identifying trade-offs can be challenging owing to the fact that individuals often vary in resource acquisition and energy budget. Because individuals with larger energy budgets will tend to expend more on all functions compared to less well-nourished individuals, this can lead to positive correlations between different classes of expenditure when groups of individuals are compared. It is possible that such "phenotypic correlations" (Hill and Hurtado, 1996) help explain some of our unanticipated findings, such as the positive association between testosterone and measures of immunity (Gettler et al., 2014a). More generally, physiological trade-offs that evolved under ancestral conditions of resource scarcity, which are mediated by adaptive patterns of genetic pleiotropy (Finch and Rose, 1995), may be less easy to identify when studied under contemporary conditions of chronic energy excess.

Life history theory focuses on functional trade-offs, and thus has greatest applicability when studying phenotypes with clear functional significance. Because many disease risk factors 
and processes studied using cohort designs have ambiguous functions, it is often not straightforward to link such measures to theoretical expectations from LHT. Thus, it is rarely simple to integrate studies of widely-studied pathological processes, such as elevated LDL cholesterol or hypertension, into LHT inspired hypotheses. Future work demonstrating the functional significance of such measures, inspired for instance by their role in support of cellular turnover, metabolism or growth (Kuzawa et al., 2006), could help broaden the applicability of theoretical predictions from evolutionary frameworks like life history theory. These limitations aside, we feel that CLHNS work that has been motivated by LHT helps illustrate the power of this integrative framework to inspire testable hypotheses addressing key domains of human population biological research, including growth, reproduction, behavior and aging.

Acknowledgements. The CLHNS is a long-running study that has involved numerous institutions and collaborators. We first and foremost thank three generations of study participants for their willingness to contribute to the study since its inception nearly 4 decades ago. We thank Barry Popkin, Wilhelm Flieger and the other researchers responsible for designing the baseline and original longitudinal study. We thank Karen Mohlke for her role in bringing the study of genetic and related processes to the study. We also thank the many interviewers, project staff, lab technicians, volunteers and co-authors, who are too numerous to list here. We thank the National Science Foundation, the Wenner Gren Foundation, and the National Institutes of Health for funding the research questions reviewed here: National Science Foundation (Biological Anthropology): BCS 0542182 (Lampl/Kuzawa), BCS 0746320 (Kuzawa), BCS 0746320 (Kuzawa/Quinn), BCS 0962212 (Kuzawa/Gettler), BCS 0962282 (Kuzawa/Eisenberg), BCS 1317133 (Kuzawa), BCS-1440564 (McDade), BCS-1519110 (Eisenberg), BCS 1751912 
(Kuzawa/Ryan); Wenner Gren Foundation: Gr. 7356 (Kuzawa), Gr. 8111 (Eisenberg), Gr.

8186 (Gettler); National Institutes of Health: R01 AG039443 (Adair), R01 HD054501 (Adair), R03 HD062715 (Kuzawa/Rutherford) RO1 HL085144 (Adair), R01 AG061006 (Kuzawa). 
References Cited 

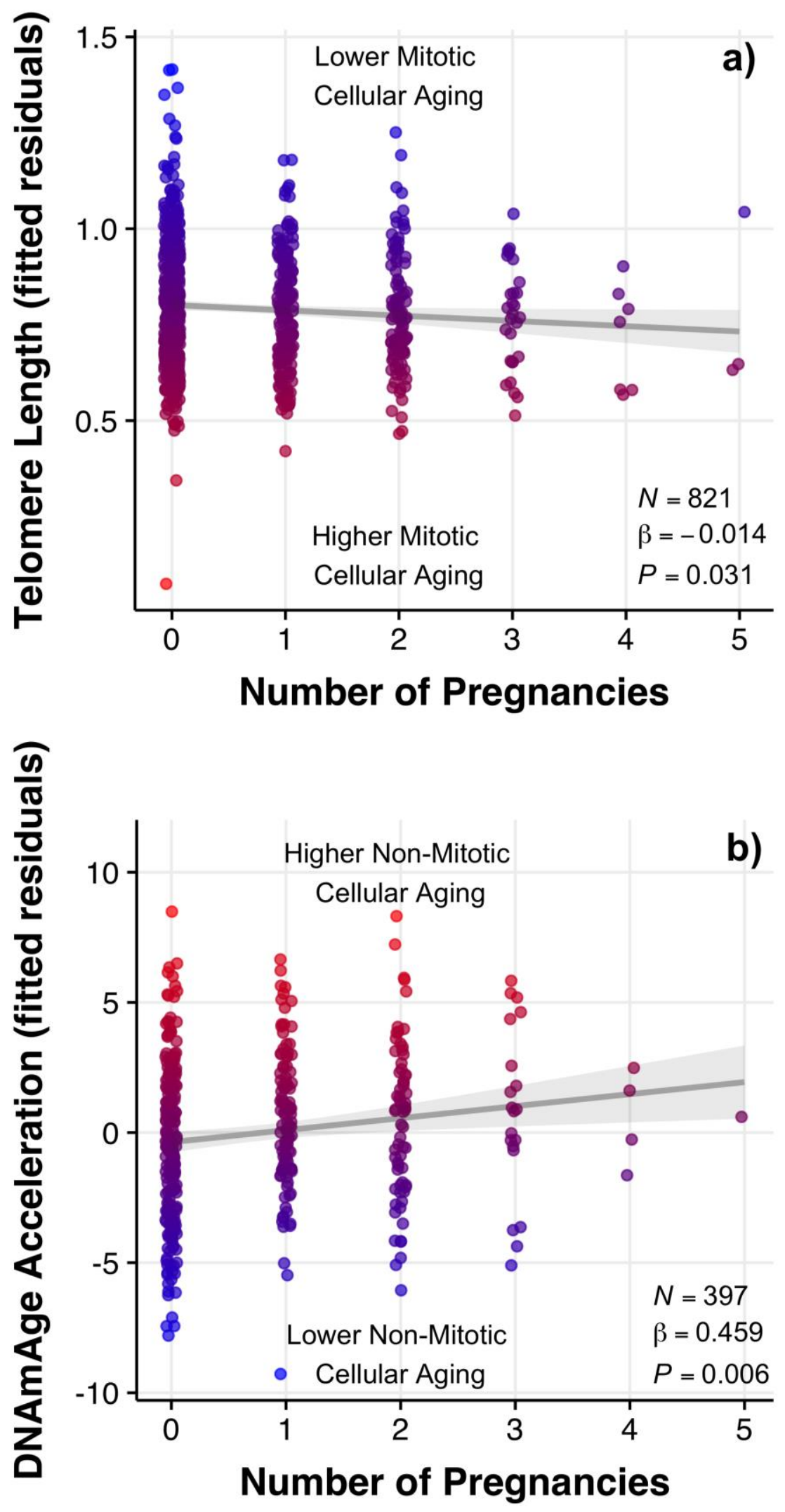
Fig. 1. Evidence for costs of reproduction in 20-22 year old female participants in the Cebu Longitudinal Health and Nutrition Survey. Figure reprinted with permission from Ryan et al 2018. 

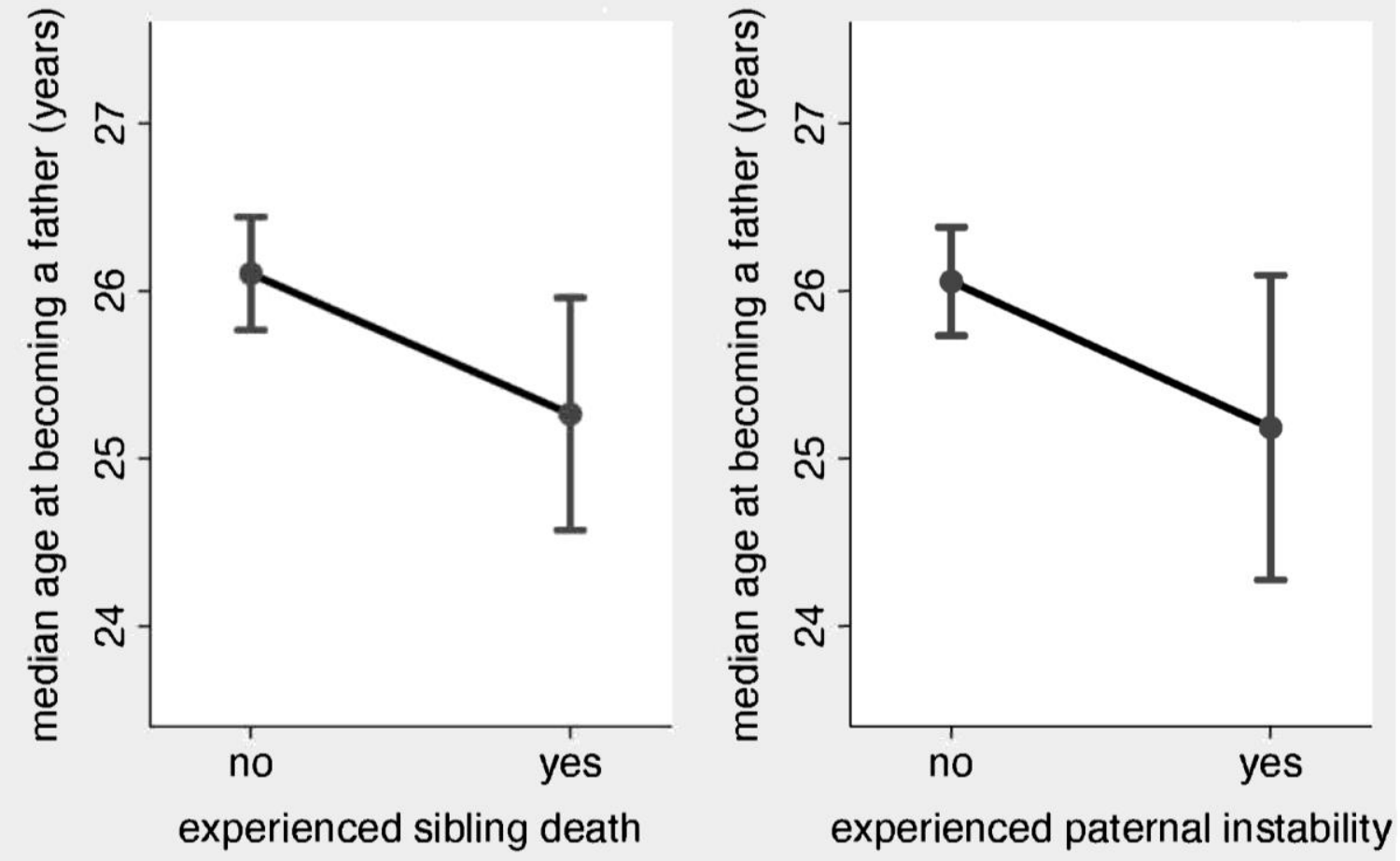

Fig. 2. Early cues of mortality and familial instability predict an earlier transition to fatherhood among 21-23 year old male participants in the Cebu Longitudinal Health and Nutrition Survey. Figure reprinted with permission from Gettler et al 2015. 


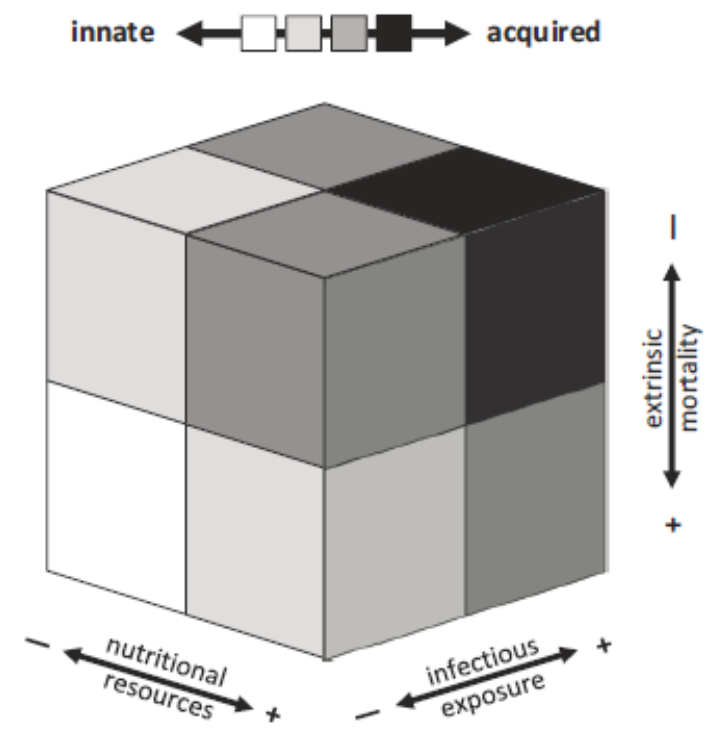

Fig 3. Higher levels of nutritional resources, more intense pathogen exposure, and lower extrinsic mortality risk should promote higher levels of investment in acquired immune defenses (darker gray to black). Lower levels of nutritional resources and infectious exposures, and higher extrinsic mortality, will promote more investment in innate immunity (lighter gray to white). Absent from view is the condition with low nutritional resources, high infectious exposure, and high extrinsic mortality, which should bias investment toward innate immunity (lighter gray) (figure reprinted with permission from McDade et al 2016).

Adair, LS 2001. Size at birth predicts age at menarche. Pediatrics, 107, E59.

Adair, LS, Kuzawa, CW \& Borja, J 2001. Maternal energy stores and diet composition during pregnancy program adolescent blood pressure. Circulation, 104, 1034-9.

Adair, LS, Popkin, BM, Akin, JS, Guilkey, DK, Gultiano, S, Borja, J, Perez, L, Kuzawa, CW, Mcdade, T \& Hindin, MJ 2011. Cohort profile: the Cebu longitudinal health and nutrition survey. Int J Epidemiol, 40, 619-25.

Alvergne, A, Faurie, C \& Raymond, M 2009. Variation in testosterone levels and male reproductive effort: Insight from a polygynous human population. Hormones and Behavior, 56, 491-497.

Anderson, J, Mckinley, K, Onugha, J, Duazo, P, Chernoff, M \& Quinn, EA 2016. Lower levels of human milk adiponectin predict offspring weight for age: a study in a lean population of Filipinos. Matern Child Nutr, 12, 790-800.

Anderson, KG, Kaplan, H \& Lancaster, JB 2007. Confidence of paternity, divorce, and investment in children by Albuquerque men. Evolution and Human Behavior, 28, 1-10.

Arifeen, SE, Ekstrom, EC, Frongillo, EA, Hamadani, J, Khan, AI, Naved, RT, Rahman, A, Raqib, R, Rasmussen, KM, Selling, KE, Wagatsuma, Y \& Persson, LA 2018. Cohort 
Profile: The Maternal and Infant Nutrition Interventions in Matlab (MINIMat) cohort in Bangladesh. Int J Epidemiol, 47, 1737-1738e.

Barker, DJ 1990. The fetal and infant origins of adult disease. BMJ, 301, 1111.

Belsky, J, Steinberg, L \& Draper, P 1991. Childhood experience, interpersonal development, and reproductive strategy: An evolutionary theory of socialization. Child development, 62, 647-670.

Bouwhuis, S, Charmantier, A, Verhulst, S \& Sheldon, BC 2010. Individual variation in rates of senescence: natal origin effects and disposable soma in a wild bird population. J Anim Ecol, 79, 1251-61.

Boyette, A, Lew-Levy, S \& Gettler, L 2018. Dimensions of fatherhood in a Congo Basin village: a multi-method analysis of intra-cultural variation in men's parenting and its relevance for child health. Current Anthropology, 1-33.

Bragg, JM, Kuzawa, CW, Agustin, SS, Banerjee, MN \& Mcdade, TW 2012. Age at menarche and parity are independently associated with Anti-Mullerian hormone, a marker of ovarian reserve, in Filipino young adult women. Am J Hum Biol, 24, 739-45.

Bribiescas, RG 2006. Men - evolutionary and life history, Cambridge, Mass., Harvard University Press.

Cameron, N, De Wet, T, Ellison, GT \& Bogin, B 1998. Growth in height and weight of South African urban infants from birth to five years: The Birth to Ten Study. Am J Hum Biol, 10, 495-504.

Charnov, EL 1991. Evolution of life history variation among female mammals. Proc Natl Acad Sci U S A, 88, 1134-7.

Doblhammer, G \& Oeppen, J 2003. Reproduction and longevity among the British peerage: the effect of frailty and health selection. Proc Biol Sci, 270, 1541-7.

Eisenberg, D, Rej, P, Duazo, P, Carba, D, Hayes, MG \& Kuzawa, CW in press. Testing for paternal influences on offspring telomere length in a human cohort in the Philippines. Am $J$ Phys Anthropol.

Eisenberg, DT 2011. An evolutionary review of human telomere biology: the thrifty telomere hypothesis and notes on potential adaptive paternal effects. Am J Hum Biol, 23, 149-67.

Eisenberg, DT, Hayes, MG \& Kuzawa, CW 2012. Delayed paternal age of reproduction in humans is associated with longer telomeres across two generations of descendants. Proc Natl Acad Sci U S A, 109, 10251-6.

Eisenberg, DTA \& Kuzawa, CW 2018. The paternal age at conception effect on offspring telomere length: mechanistic, comparative and adaptive perspectives. Philos Trans $R$ Soc Lond B Biol Sci, 373.

Eisenberg, DTA, Lee, NR, Rej, PH, Hayes, MG \& Kuzawa, CW 2019. Older paternal ages and grandpaternal ages at conception predict longer telomeres in human descendants. Proc Biol Sci, 286, 20190800.

Ellis, BJ, Figueredo, AJ, Brumbach, BH \& Schlomer, GL 2009. Fundamental dimensions of environmental risk. Human Nature, 20, 204-268.

Eveleth, PB \& Tanner, JM 1990. Worldwide variation in human growth, Cambridge England ; New York, Cambridge University Press.

Finch, CE \& Rose, MR 1995. Hormones and the physiological architecture of life history evolution. $Q$ Rev Biol, 70, 1-52.

Fried, RL, Mayol, NL, Mcdade, TW \& Kuzawa, CW 2017. Maternal metabolic adaptations to pregnancy among young women in Cebu, Philippines. Am J Hum Biol, 29. 
Georgiev, AV, Kuzawa, CW \& Mcdade, TW 2016. Early developmental exposures shape tradeoffs between acquired and innate immunity in humans. Evol Med Public Health, 2016, 256-69.

Georgiev, AV, Ryan, CP, Borja, JB \& Kuzawa, CW 2017. Is early postnatal growth velocity, a proxy of minipubertal androgen action, related to adult second-to-fourth digit (2D:4D) ratios in men? A test in Cebu, Philippines. Am J Hum Biol, 29.

Gettler, LT 2016. Becoming DADS: considering the role of cultural context and developmental plasticity for paternal socioendocrinology. Current Anthropology, 57, S38-S51.

Gettler, LT, Agustin, SS \& Kuzawa, CW 2010. Testosterone, physical activity, and somatic outcomes among Filipino males. Am J Phys Anthropol, 142, 590-9.

Gettler, LT, Kuo, PX, Rosenbaum, S, Avila, JL, Mcdade, TW \& Kuzawa, CW 2019. Sociosexuality, testosterone, and life history status: prospective associations and longitudinal changes among men in Cebu, Philippines. Evolution and Human Behavior, 40, 249-258.

Gettler, LT, Mcdade, TW, Agustin, SS, Feranil, AB \& Kuzawa, CW 2013. Do testosterone declines during the transition to marriage and fatherhood relate to men's sexual behavior? Evidence from the Philippines. Horm Behav, 64, 755-63.

Gettler, LT, Mcdade, TW, Agustin, SS, Feranil, AB \& Kuzawa, CW 2014a. Testosterone, immune function, and life history transitions in Filipino males (Homo sapiens). International Journal of Primatology, 35, 787-804.

Gettler, LT, Mcdade, TW, Bragg, JM, Feranil, AB \& Kuzawa, CW 2015. Developmental energetics, sibling death, and parental instability as predictors of maturational tempo and life history scheduling in males from Cebu, Philippines. Am J Phys Anthropol, 158, 175184.

Gettler, LT, Mcdade, TW, Feranil, AB, Agustin, SS \& Kuzawa, CW 2014b. Salivary estradiol and testosterone in Filipino men: Diurnal patterns and relationships with adiposity. Am J Hum Biol, 26, 376-83.

Gettler, LT, Mcdade, TW, Feranil, AB \& Kuzawa, CW 2011a. Longitudinal evidence that fatherhood decreases testosterone in human males. Proc Natl Acad Sci U S A, 108, 16194-9.

Gettler, LT, Mcdade, TW, Feranil, AB \& Kuzawa, CW 2012a. Prolactin, fatherhood, and reproductive behavior in human males. Am J Phys Anthropol, 148, 362-70.

Gettler, LT, Mcdade, TW \& Kuzawa, CW 2011b. Cortisol and testosterone in Filipino young adult men: evidence for co-regulation of both hormones by fatherhood and relationship status. Am J Hum Biol, 23, 609-20.

Gettler, LT, Mckenna, JJ, Mcdade, TW, Agustin, SS \& Kuzawa, CW 2012b. Does cosleeping contribute to lower testosterone levels in fathers? Evidence from the Philippines. PLoS One, 7, e41559.

Gettler, LT, Ryan, CP, Eisenberg, DTA, Rzhetskaya, M, Hayes, MG, Feranil, AB, Bechayda, SA \& Kuzawa, CW 2017. The role of testosterone in coordinating male life history strategies: The moderating effects of the androgen receptor CAG repeat polymorphism. Horm Behav, 87, 164-175.

Gluckman, PD \& Hanson, MA 2006. Evolution, development and timing of puberty. Trends Endocrinol Metab, 17, 7-12.

Gray, PB \& Anderson, KG 2010. Fatherhood: Evolution and Human Paternal Behavior, Cambridge, MA, Harvard University Press. 
Gray, PB, Kahlenberg, SM, Barrett, ES, Lipson, SF \& Ellison, PT 2002. Marriage and fatherhood are associated with lower testosterone in males. Evolution and Human Behavior, 23, 193-201.

Gray, PB, Mchale, TS \& Carré, JM 2017. A review of human male field studies of hormones and behavioral reproductive effort. Hormones and Behavior, 91, 52-67.

Grebe, NM, Sarafin, RE, Strenth, CR \& Zilioli, S 2019. Pair-bonding, fatherhood, and the role of testosterone: A meta-analytic review. Neuroscience \& Biobehavioral Reviews, 98, 221233.

Hanson, M \& Gluckman, P 2011. Developmental origins of noncommunicable disease: population and public health implications. Am J Clin Nutr, 94, 1754S-1758S.

Hennig, BJ, Unger, SA, Dondeh, BL, Hassan, J, Hawkesworth, S, Jarjou, L, Jones, KS, Moore, SE, Nabwera, HM, Ngum, M, Prentice, A, Sonko, B, Prentice, AM \& Fulford, AJ 2017. Cohort Profile: The Kiang West Longitudinal Population Study (KWLPS)-a platform for integrated research and health care provision in rural Gambia. Int J Epidemiol, 46, e13.

Hewlett, BS 1991. Intimate fathers: The nature and context of Aka Pygmy paternal infant care, Ann Arbor, University of Michigan Press.

Hewlett, BS \& Macfarlan, SJ 2010. Fathers' roles in hunter-gatherer and other small-scale cultures. In: Lamb, M. E. (ed.) The Role of the Father in Child Development. Hoboken, NJ: John Wiley \& Sons, Inc.

Hill, K \& Hurtado, AM 1996. Aché life history : the ecology and demography of a foraging people, New York, Aldine de Gruyter.

Hill, K \& Kaplan, H 1999. Life history traits in humans: Theory and empirical studies. Annual review of anthropology, 28, 397-430.

Ho, KK, Pinsky, JL, Kannel, WB \& Levy, D 1993. The epidemiology of heart failure: the Framingham Study. J Am Coll Cardiol, 22, 6A-13A.

Jasienska, G 2009. Reproduction and lifespan: Trade-offs, overall energy budgets, intergenerational costs, and costs neglected by research. Am J Hum Biol, 21, 524-32.

Kirkwood, TB \& Rose, MR 1991. Evolution of senescence: late survival sacrificed for reproduction. Philos Trans $R$ Soc Lond B Biol Sci, 332, 15-24.

Kleiber, M 1932. Body size and metabolism. Hilgardia, 6, 315-353.

Kleiman, DG \& Malcolm, JR 1981. The evolution of male parental investment in mammals. In: Gubernick, D. \& Klopfer, P. (eds.) Parental Care in Mammals. New York, NY: Plenum Press.

Kuzawa, C, Snodgrass, J \& Leonard, W. Year. Evolutionary medicine and human cholesterol. In: AMERICAN JOURNAL OF HUMAN BIOLOGY, 2006. WILEY-LISS DIV JOHN WILEY \& SONS INC, 111 RIVER ST, HOBOKEN, NJ 07030 USA, 261-261.

Kuzawa, CW 2005. Fetal origins of developmental plasticity: are fetal cues reliable predictors of future nutritional environments? Am J Hum Biol, 17, 5-21.

Kuzawa, CW 2007. Developmental origins of life history: growth, productivity, and reproduction. Am J Hum Biol, 19, 654-61.

Kuzawa, CW \& Adair, LS 2003. Lipid profiles in adolescent Filipinos: relation to birth weight and maternal energy status during pregnancy. Am J Clin Nutr, 77, 960-6.

Kuzawa, CW \& Bragg, JM 2012. Plasticity in human life history strategy: Implications for contemporary human variation and the evolution of genus Homo. Current Anthropology, 53, S369-S382. 
Kuzawa, CW, Chugani, HT, Grossman, LI, Lipovich, L, Muzik, O, Hof, PR, Wildman, DE, Sherwood, CC, Leonard, WR \& Lange, N 2014. Metabolic costs and evolutionary implications of human brain development. Proc Natl Acad Sci U S A, 111, 13010-5.

Kuzawa, CW, Fried, RL, Borja, JB \& Mcdade, TW 2017. Maternal pregnancy C-reactive protein predicts offspring birth size and body composition in metropolitan Cebu, Philippines. $J$ Dev Orig Health Dis, 8, 674-681.

Kuzawa, CW, Gettler, LT, Huang, Y-Y \& Mcdade, TW 2010a. Mothers have lower testosterone than non-mothers: Evidence from the Philippines. Hormones and Behavior, 57, 441-447.

Kuzawa, CW, Gettler, LT, Muller, MN, Mcdade, TW \& Feranil, AB 2009. Fatherhood, pairbonding and testosterone in the Philippines. Horm Behav, 56, 429-35.

Kuzawa, CW, Mcdade, TW, Adair, LS \& Lee, N 2010b. Rapid weight gain after birth predicts life history and reproductive strategy in Filipino males. Proc Natl Acad Sci U S A, 107, 16800-5.

Kuzawa, CW \& Quinn, EA 2009. Developmental origins of adult function and health: evolutionary hypotheses. Annual Review of Anthropology, 38, 131-147.

Kyweluk, MA, Georgiev, AV, Borja, JB, Gettler, LT \& Kuzawa, CW 2018. Menarcheal timing is accelerated by favorable nutrition but unrelated to developmental cues of mortality or familial instability in Cebu, Philippines. Evolution and Human Behavior, 39, 76-81.

Lamb, ME 2004. The role of the father in child development, John Wiley \& Sons.

Lee, J, Fried, R, Thayer, Z \& Kuzawa, CW 2014. Preterm delivery as a predictor of diurnal cortisol profiles in adulthood: evidence from Cebu, Philippines. Am J Hum Biol, 26, 598602.

Lukas, D \& Clutton-Brock, TH 2013. The evolution of social monogamy in mammals. Science, 341, 526-30.

Marlowe, F 2000. Paternal investment and the human mating system. Behavioural Processes, 51, 45-61.

Marmot, MG, Smith, GD, Stansfeld, S, Patel, C, North, F, Head, J, White, I, Brunner, E \& Feeney, A 1991. Health inequalities among British civil servants: the Whitehall II study. Lancet, 337, 1387-93.

Mattison, SM, Scelza, B \& Blumenfield, T 2014. Paternal Investment and the Positive Effects of Fathers among the Matrilineal Mosuo of Southwest China. American Anthropologist, $116,591-610$.

Mcdade, TW 2003. Life history theory and the immune system: steps toward a human ecological immunology. Am J Phys Anthropol, Suppl 37, 100-25.

Mcdade, TW 2005. Life history, maintenance, and the early origins of immune function. Am J Hum Biol, 17, 81-94.

Mcdade, TW, Beck, MA, Kuzawa, CW \& Adair, LS 2001. Prenatal undernutrition and postnatal growth are associated with adolescent thymic function. $J$ Nutr, 131, 1225-31.

Mcdade, TW, Georgiev, AV \& Kuzawa, CW 2016. Trade-offs between acquired and innate immune defenses in humans. Evol Med Public Health, 2016, 1-16.

Mcdade, TW, Jones, MJ, Miller, G, Borja, J, Kobor, MS \& Kuzawa, CW 2018. Birth weight and postnatal microbial exposures predict the distribution of peripheral blood leukocyte subsets in young adults in the Philippines. J Dev Orig Health Dis, 9, 198-207.

Mcdade, TW, Rutherford, J, Adair, L \& Kuzawa, CW 2010. Early origins of inflammation: microbial exposures in infancy predict lower levels of C-reactive protein in adulthood. Proc Biol Sci, 277, 1129-37. 
Mcdade, TW, Ryan, C, Jones, MJ, Macisaac, JL, Morin, AM, Meyer, JM, Borja, JB, Miller, GE, Kobor, MS \& Kuzawa, CW 2017. Social and physical environments early in development predict DNA methylation of inflammatory genes in young adulthood. Proc Natl Acad Sci U S A, 114, 7611-7616.

Mitteldorf, J 2010. Female fertility and longevity. Age (Dordr), 32, 79-84.

Muehlenbein, MP \& Bribiescas, RG 2005. Testosterone-mediated immune functions and male life histories. Am J Hum Biol, 17, 527-58.

Muller, MN 2017. Testosterone and reproductive effort in male primates. Hormones and Behavior, 91, 36-51.

Nettle, D, Coall, DA \& Dickins, TE 2011. Early-life conditions and age at first pregnancy in British women. Proc Biol Sci, 278, 1721-7.

Poulton, R, Moffitt, TE \& Silva, PA 2015. The Dunedin Multidisciplinary Health and Development Study: overview of the first 40 years, with an eye to the future. Soc Psychiatry Psychiatr Epidemiol, 50, 679-93.

Quinn, EA 2013. No evidence for sex biases in milk macronutrients, energy, or breastfeeding frequency in a sample of Filipino mothers. Am J Phys Anthropol, 152, 209-16.

Quinn, EA, Largado, F, Borja, JB \& Kuzawa, CW 2015. Maternal characteristics associated with milk leptin content in a sample of Filipino women and associations with infant weight for age. J Hum Lact, 31, 273-81.

Richter, L, Norris, S, Pettifor, J, Yach, D \& Cameron, N 2007. Cohort Profile: Mandela's children: the 1990 Birth to Twenty study in South Africa. Int J Epidemiol, 36, 504-11.

Rosenbaum, S, Gettler, LT, Mcdade, TW, Bechayda, SS \& Kuzawa, CW 2018. Does a man's testosterone "rebound" as dependent children grow up, or when pairbonds end? A test in Cebu, Philippines. Am J Hum Biol, 30, e23180.

Rutherford, JN, Victoria, AD, Ragsdale, HB, Avila, JL, Lee, NR \& Kuzawa, CW 2019. Global population variation in placental size and structure: Evidence from $\mathrm{Cebu}$, Philippines. Placenta, 85, 40-48.

Ryan, CP, Hayes, MG, Lee, NR, Mcdade, TW, Jones, MJ, Kobor, MS, Kuzawa, CW \& Eisenberg, DTA 2018. Reproduction predicts shorter telomeres and epigenetic age acceleration among young adult women. Sci Rep, 8, 11100.

Ryan, CP, Mcdade, TW, Gettler, LT, Eisenberg, DT, Rzhetskaya, M, Hayes, MG \& Kuzawa, CW 2017. Androgen receptor CAG repeat polymorphism and hypothalamic-pituitarygonadal function in Filipino young adult males. Am J Hum Biol, 29.

Sarma, MS, Kuo, PX, Bechayda, SA, Kuzawa, CW \& Gettler, LT 2018. Exploring the links between early life and young adulthood social experiences and men's later life psychobiology as fathers. Physiol Behav, 193, 82-89.

Schooling, CM, Jiang, CQ, Heys, M, Zhang, WS, Lao, XQ, Adab, P, Cowling, BJ, Thomas, GN, Cheng, KK, Lam, TH \& Leung, GM 2008. Is leg length a biomarker of childhood conditions in older Chinese women? The Guangzhou Biobank Cohort Study. J Epidemiol Community Health, 62, 160-6.

Stearns, SC 1992. The evolution of life histories, Oxford ; New York, Oxford University Press.

Stearns, SC \& Koella, JC 1986. The evolution of phenotypic plasticity in life-history traits: predictions of reaction norms for age and size at maturity. Evolution, 40, 893-913.

Stearns, SC \& Rodrigues, AMM in press. On the use of "life history theory" in evolutionary psychology. Evol Hum Behav. 
Stein, AD, Melgar, P, Hoddinott, J \& Martorell, R 2008. Cohort Profile: the Institute of Nutrition of Central America and Panama (INCAP) Nutrition Trial Cohort Study. Int J Epidemiol, 37, 716-20.

Thayer, ZM, Feranil, AB \& Kuzawa, CW 2012. Maternal cortisol disproportionately impacts fetal growth in male offspring: evidence from the Philippines. Am J Hum Biol, 24, 1-4.

Urlacher, SS, Snodgrass, JJ, Dugas, LR, Sugiyama, LS, Liebert, MA, Joyce, CJ \& Pontzer, H 2019. Constraint and trade-offs regulate energy expenditure during childhood. Sci Adv, 5, eaax 1065 .

Walker, SP, Chang, SM, Powell, CA \& Grantham-Mcgregor, SM 2005. Effects of early childhood psychosocial stimulation and nutritional supplementation on cognition and education in growth-stunted Jamaican children: prospective cohort study. Lancet, 366, 1804-7.

West, GB, Brown, JH \& Enquist, BJ 1999. The fourth dimension of life: fractal geometry and allometric scaling of organisms. Science, 284, 1677-9.

West, GB, Woodruff, WH \& Brown, JH 2002. Allometric scaling of metabolic rate from molecules and mitochondria to cells and mammals. Proc Natl Acad Sci U S A, 99 Suppl 1, 2473-8.

Westendorp, RG \& Kirkwood, TB 1998. Human longevity at the cost of reproductive success. Nature, 396, 743-6.

Williams, GC 1966. Adaptation and natural selection; a critique of some current evolutionary thought, Princeton, N.J.,, Princeton University Press.

Wilson, M \& Daly, M 1997. Life expectancy, economic inequality, homicide, and reproductive timing in Chicago neighbourhoods. BMJ, 314, 1271-4.

Wingfield, J, Hegner, R, Ball, G \& Duffy, A 1990. The 'challenge hypothesis': theoretical implications for patterns of testosterone secretion, mating systems, and breeding strategies. Am Nat, 136.

Wingfield, JC 2017. The challenge hypothesis: Where it began and relevance to humans. Hormones and Behavior, 92, 9-12.

Winking, J, Gurven, M, Kaplan, H \& Stieglitz, J 2009. The goals of direct paternal care among a South Amerindian population. American Journal of Physical Anthropology, 139, 295304. 